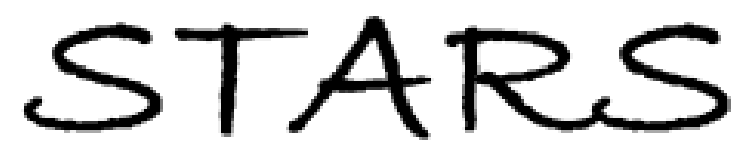

University of Central Florida

STARS

$1-1-2005$

\title{
Scattering of g-process longitudinal optical phonons at hotspots in silicon
}

\author{
S. Sinha \\ P. K. Schelling \\ University of Central Florida \\ S. R. Phillpot \\ K. E. Goodson
}

Find similar works at: https://stars.library.ucf.edu/facultybib2000

University of Central Florida Libraries http://library.ucf.edu

This Article is brought to you for free and open access by the Faculty Bibliography at STARS. It has been accepted for inclusion in Faculty Bibliography 2000s by an authorized administrator of STARS. For more information, please contactSTARS@ucf.edu.

\section{Recommended Citation}

Sinha, S.; Schelling, P. K.; Phillpot, S. R.; and Goodson, K. E., "Scattering of g-process longitudinal optical phonons at hotspots in silicon" (2005). Faculty Bibliography 2000s. 5678.

https://stars.library.ucf.edu/facultybib2000/5678

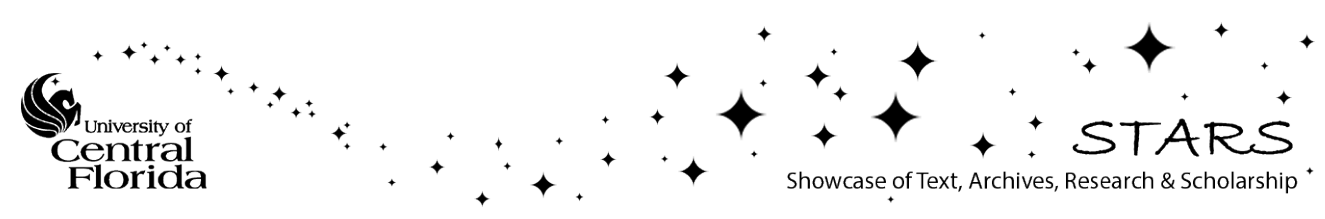




\section{Scattering of $g$-process longitudinal optical phonons at hotspots in silicon}

Cite as: J. Appl. Phys. 97, 023702 (2005); https://doi.org/10.1063/1.1831549

Submitted: 13 April 2004 . Accepted: 18 October 2004 . Published Online: 23 December 2004

S. Sinha, P. K. Schelling, S. R. Phillpot, and K. E. Goodson

\section{ARTICLES YOU MAY BE INTERESTED IN}

Nanoscale thermal transport

Journal of Applied Physics 93, 793 (2003); https://doi.org/10.1063/1.1524305

Nanoscale thermal transport. II. 2003-2012

Applied Physics Reviews 1, 011305 (2014); https://doi.org/10.1063/1.4832615

Phonon wave-packet dynamics at semiconductor interfaces by molecular-dynamics simulation

Applied Physics Letters 80, 2484 (2002); https://doi.org/10.1063/1.1465106

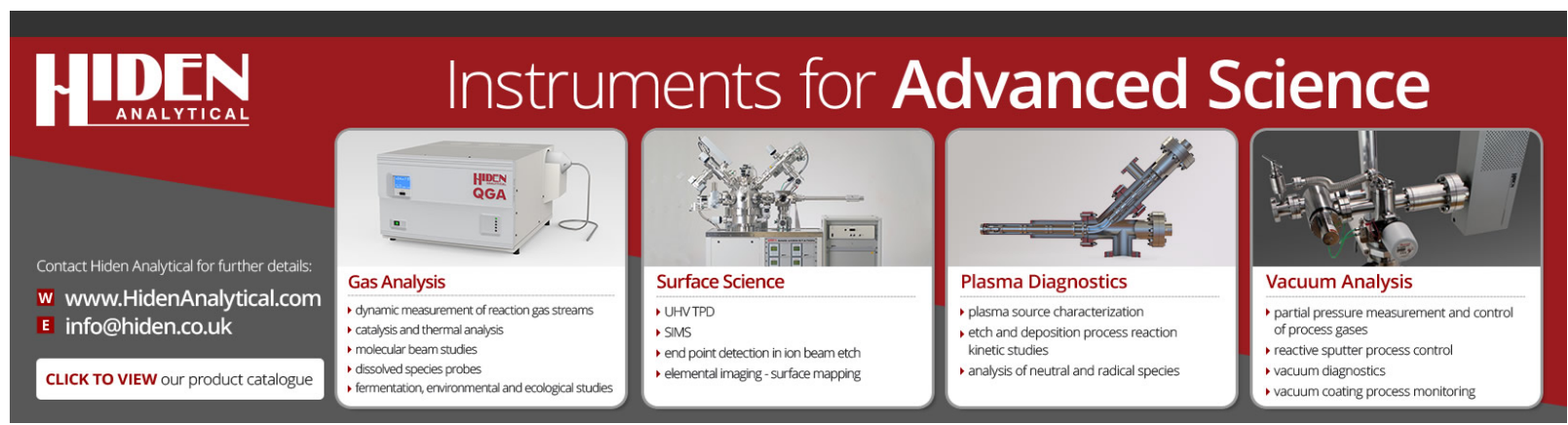




\title{
Scattering of $\boldsymbol{g}$-process longitudinal optical phonons at hotspots in silicon
}

\author{
S. Sinha ${ }^{a)}$ \\ Thermosciences Division, Mechanical Engineering Department, Stanford University, Stanford, \\ California 94305-3030 \\ P. K. Schelling \\ Materials Science Division, Argonne National Laboratory, Argonne, Illinois 60439, and \\ Advanced Materials Processing and Analysis Center (AMPAC), and Department of Physics, \\ University of Central Florida, Orlando, Florida 32816
}

S. R. Phillpot

Materials Science Division, Argonne National Laboratory, Argonne, Illinois 60439, and Department of Materials Science and Engineering, University of Florida, Gainesville, Florida 32611

\section{K. E. Goodson}

Thermosciences Division, Mechanical Engineering Department, Stanford University, Stanford, California 94305-3030

(Received 13 April 2004; accepted 18 October 2004; published online 23 December 2004)

\begin{abstract}
Transistors with gate lengths below $100 \mathrm{~nm}$ generate phonon hotspots with dimensions on the order of $10 \mathrm{~nm}$ and peak power densities of about $50 \mathrm{~W} / \mu \mathrm{m}^{3}$. This work employs molecular dynamics to investigate the impact of lattice energy density on phonon scattering at the hotspot. The hotspot studied in this work consists of longitudinal optical phonons involved in the $g$-type intervalley scattering of conduction electrons in silicon. A comparison of the decay modes in hotspots with high and moderate energy densities reveals that the decay mechanisms are the same but the relaxation rates differ. Scattering occurs through a three phonon process of the form $L O \rightarrow L A+T A$, involving the zone-edge transverse acoustic modes. An increase in the energy density from a moderate value of 5 to $125 \mathrm{~W} / \mu \mathrm{m}^{3}$ changes the relaxation time from 79 to $16 \mathrm{ps}$, approximately proportional to the the maximum initial amplitude of the phonons. This work improves the accuracy of the scattering rates of optical phonons and helps in advancing the electro-thermal modeling of nanotransistors. () 2005 American Institute of Physics. [DOI: 10.1063/1.1831549]
\end{abstract}

\section{INTRODUCTION}

Nonequilibrium among phonon modes ${ }^{1}$ in a silicon metal-oxide-semiconductor field-effect transistor impedes heat conduction as the device feature size is scaled to below $100 \mathrm{~nm}$. Most of the phonon generation occurs over a length scale of $10 \mathrm{~nm}$ at the drain terminal of the device, resulting in nanoscale hotspots as shown in Fig. 1. There is a local increase in the thermal resistance at the drain, which can directly affect the $I-V$ characteristics. ${ }^{2}$ In nanotransistors the problem is worsened by the increased power density, ${ }^{3}$ which may be as high as $50 \mathrm{~W} / \mu^{3}$. Several numerical ${ }^{4,5}$ and compact ${ }^{6,7}$ models based on moments of the phonon Boltzmann transport equation (BTE) have been proposed to predict the increase in the thermal resistance at the hotspot. The models show that hot phonon modes remain in nonequilibrium over a large distance from the hotspot thereby reducing the effective conductance. This radiative effect is significant only when the size of the hotspot is small compared to the the mean free path of thermally excited phonons outside the hotspot. ${ }^{6}$ Thermometry ${ }^{8}$ at a hotspot in thin-film silicon shows that the thermal resistance exceeds predictions based on the Fourier law by $60 \%$ when the phonon mean free path is a factor of 30 larger than the size of the hotspot. Since the

\footnotetext{
${ }^{a)}$ Author to whom correspondence should be addressed; electronic mail: sanjiv@stanford.edu
}

hotspot in a bulk silicon device is a similar factor smaller than the phonon mean free path at $300 \mathrm{~K}$, there would be a significant increase in the peak lattice temperature inside the device as compared to any design prediction based on the

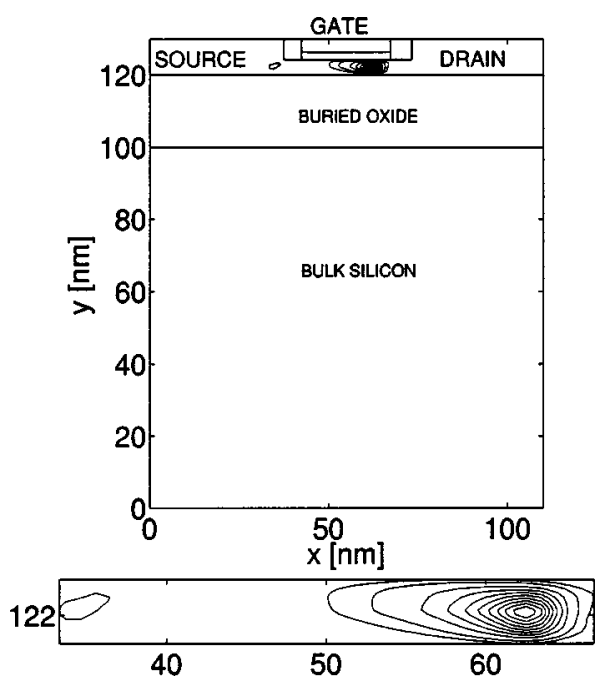

FIG. 1. The location of the hotspot in an ultrathin-body silicon-on-insulator transistor is shown. The device (see Ref. 3) has a gate length of $18 \mathrm{~nm}$. A zoomed-in view of the heat generation contours inside the channel is given at the bottom. The contours are equi-spaced at $0.5 \mathrm{~W} / \mu \mathrm{m}^{3}$ with a peak value of $65 \mathrm{~W} / \mu \mathrm{m}^{3}$ at the center. Device simulations were performed using the commercial MEDICI software. 
heat diffusion equation. An increase in the lattice temperature inside the channel region can reduce the drain current significantly. Coupled electro-thermal simulations based on a hydrodynamic model for electrons and phonons ${ }^{2,9}$ show that the drain current is reduced by $25 \%$ when the lattice temperature is increased by $100 \mathrm{~K}$ in a GaAs device.

In addition to the size of the hotspot, the high population of low group velocity optical phonons at the hotspot also reduces heat conduction from the hotspot. Heat conduction is impeded until the low group velocity optical modes decay into higher group velocity acoustic modes. This aspect has not been investigated in detail: either experimentally or theoretically, in a device. Though there is considerable literature on phonon decay in optically excited hotspots in general (see Ref. 10, for example), these low temperature studies have focused almost exclusively on zone-center optical phonons, that are characterized by low electron-phonon scattering rates in silicon, and hence are unlikely to be generated in the hotspot of a transistor. Theoretical studies on devices, based on moments of the phonon BTE, ${ }^{2,4,5,9}$ have exaggerated the effect of the low group velocity by assuming all optical modes involved in electron-phonon scattering to have zero group velocities. However, the group velocity of some of the optical modes involved can be as high as $1500 \mathrm{~m} / \mathrm{s}$. Thus, the role of low but nonzero group velocity is not clear from these studies. Further, an approach based on the moments of the phonon BTE does not monitor details of phonon scattering from one mode to another but lumps all interactions together under an overall relaxation rate. The specifics of phonon decay are thus overlooked in such an approach. Even though the transfer of lattice energy from optical to acoustic phonons is critical to heat conduction, little is known about these anharmonic interactions under the thermal conditions prevailing in a transistor. Finally, the relaxation rates in BTE studies are borrowed from the literature on thermal conductivity theory for semiconductors. ${ }^{11,12}$ These rates, however, are derived from results of classical perturbation theory that have been fit to thermal conductivity data for the bulk material. They cannot, therefore, be expected to describe the highly nonequilibrium conditions characteristic of a hotspot.

To address the issues described above, we compute the anharmonic scattering rates for optical phonons at a hotspot, find their decay modes, and study the dependence of the rates on the energy density at the hotspot. There are two principal methods for computing anharmonic phonon scattering rates and decay modes: perturbation methods and moleculardynamics (MD) simulations. In perturbation theory, ${ }^{13,14}$ the lifetime of a phonon mode can be calculated using the cubic anharmonicity as a small perturbation to the harmonic Hamiltonian. Debernardi, Baroni, and Molinari ${ }^{15}$ have used ab initio perturbation methods to compare the decay of zone center optical phonons in carbon, silicon, and germanium. Molecular dynamics can also be used to extract phonon lifetimes by Fourier analyzing the atomic displacements and velocities at any instant to resolve various phonon modes. The MD method has been used by Fabian and Allen, ${ }^{16}$ and later by Bickham and Feldman ${ }^{17}$ to study lifetimes of vibrons (analogous to phonons) in glasses. Oligschleger and Schön ${ }^{18}$ have investigated the decay of single phonon modes in sele- nium and quartz. Ladd, Moran, and Hoover ${ }^{19}$ have compared MD calculations with classical perturbation theory calculations to show good agreement in thermal conductivity and phonon lifetimes for low-amplitude excitations.

In this work we study phonon transport and scattering near a hotspot using MD simulations. In particular, we extend the method of phonon wave packet dynamics, initially developed for thermal transport at interfaces ${ }^{20}$ and in superlattices, ${ }^{21}$ to study the evolution of a phonon hotspot. The hotspot is set up as a phonon wave packet with size, energy density, polarization, and wave vector chosen to closely match expected hotspots in a silicon device. We study the decay of the wave packets using MD simulation. We have found that inserting the hotspot into the crystal presents a considerable practical challenge. In particular, the MD supercell tends to produce a spurious strain field that propagates freely in the system and acts to destabilize the hotspot. We present a two-step procedure to eliminate this artifact. We then study phonon-phonon decay mechanisms for different energy densities in the initial hotspot. From the results of these simulations, we provide estimates of scattering rates in the absence of thermally excited phonons. The method is general enough to be extended to other studies on phonon transport and provides a powerful new tool for elucidating phonon-phonon scattering in transistors and other nanostructures.

\section{PHONON WAVE VECTOR CONSIDERATIONS IN ELECTRON-PHONON SCATTERING}

In the channel region of a transistor, electrons scatter with phonons, impurities, and surfaces. In this work we focus on scattering involving phonons. Electron-phonon scattering can be categorized as intervalley and intravalley depending on whether the scattering moves the electrons within a valley or from one valley to another in wave vector space. Intravalley scattering in silicon is entirely due to acoustic phonons since optical phonons are forbidden from energy and symmetry considerations. ${ }^{22}$ Intervalley scattering, however, involves mainly optical and zone-edge acoustic phonons. Energetic electrons tend to scatter more with optical phonons since such processes result in a higher energy loss. ${ }^{23}$ An electron must have an energy in excess of about 51 $\mathrm{meV}$, the minimum energy of optical phonons in silicon, in order to emit an optical phonon.

Phonon modes that conserve the crystal momentum in intervalley processes have been calculated by Long. ${ }^{24}$ The constant energy surface at the bottom of the conduction bands in silicon consists of six equivalent ellipsoids along the $\langle 100\rangle$ directions as shown in Fig. 2. The conduction band minima occur at about 0.85 of the distance from the zone center to the zone edge along these directions. ${ }^{25}$ Intervalley scattering requires a large change in the wave vector as shown by the arrows in Fig. 2. The two types of electron transitions, labeled $g$ - and $f$-scattering, involve scattering between valleys along the same axis and along different axes, respectively. In the reduced zone scheme, both are umklapp processes as shown in Fig 3 . The $g$ process requires a phonon with wave vector $0.3 \times(2 \pi / a)\langle 001\rangle$. The $f$ process re- 


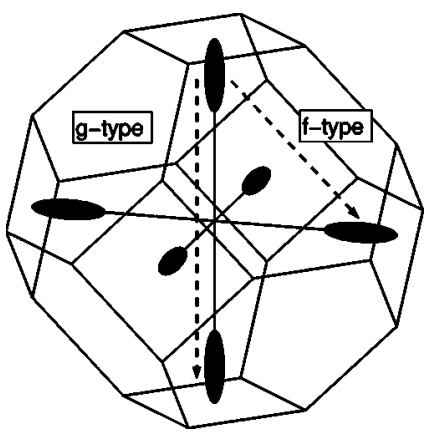

FIG. 2. The $f$ - and $g$-intervalley electronic transitions (see Ref. 24) involve optical and acoustic phonons. The present work studies the propagation and scattering of the longitudinal optical phonons that are involved in the $g$-type transition. This mode has a wave vector of $0.3[001] 2 \pi / a$.

quires a phonon with wave vector that is $11^{\circ}$ off $\langle 100\rangle$. In the sketch of Fig. 3, the wave vector is approximately $(2 \pi / a)$ $\times[\overline{0.15}, \overline{0.15}, \overline{1}]$. Further, symmetry considerations ${ }^{26}$ based on a zeroth order expansion dictate that only LO phonons are allowed in a $g$ process and LA and TO are allowed in an $f$ process. However, it has been shown by a first order expansion that low frequency TA and LA phonons are also involved in the $g$ process. ${ }^{22}$ In fact, scattering with these modes must be considered in order to match the mobility data in silicon. ${ }^{25}$ The $f$ and $g$ transition picture holds up to moderately high electric fields $\left(<\approx 10^{5} \mathrm{~V} / \mathrm{cm}\right)$. Since the fields in nanotransistors are expected to be on the order of $10^{5} \mathrm{~V} / \mathrm{cm}$, the $f$ and $g$ processes fully describe intervalley electronphonon scattering for the purpose of this article.

The hotspot in the device is predominantly populated with the optical and acoustic $f$ and $g$ phonons that are mentioned above. The $g$-type LO phonon has a group velocity of approximately $1500 \mathrm{~m} / \mathrm{s}$, whereas the $f$-type TO phonon has a zero group velocity. Acoustic phonons involved in intervalley scattering have higher group velocities. Optical modes, having higher energies, are increasingly likely to be emitted as the electrons reach the peak field region in the device. Here, the disparity in the electron and phonon energy relaxation rates, which are approximately $0.1 \mathrm{ps}$ for electrons and on the order of $10 \mathrm{ps}$ for optical phonons, ${ }^{22,27}$ coupled with strong excitation of optical phonon modes with low group velocity, results in a region of high phonon density. This is referred to as the phonon hotspot. ${ }^{10}$ We discuss the device hotspot in greater detail in the next section.

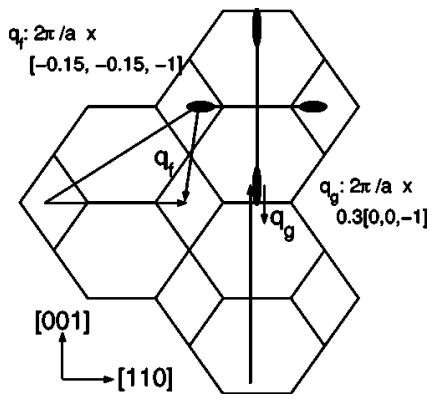

FIG. 3. The $f$ and $g$ processes are shown in a (110) plane to identify the wave vectors of the phonons involved. The $f$-process phonon has the wave vector $\mathbf{q}_{f}$ and the $g$-process phonon has the wave vector $\mathbf{q}_{g}$ purely from geometry considerations.
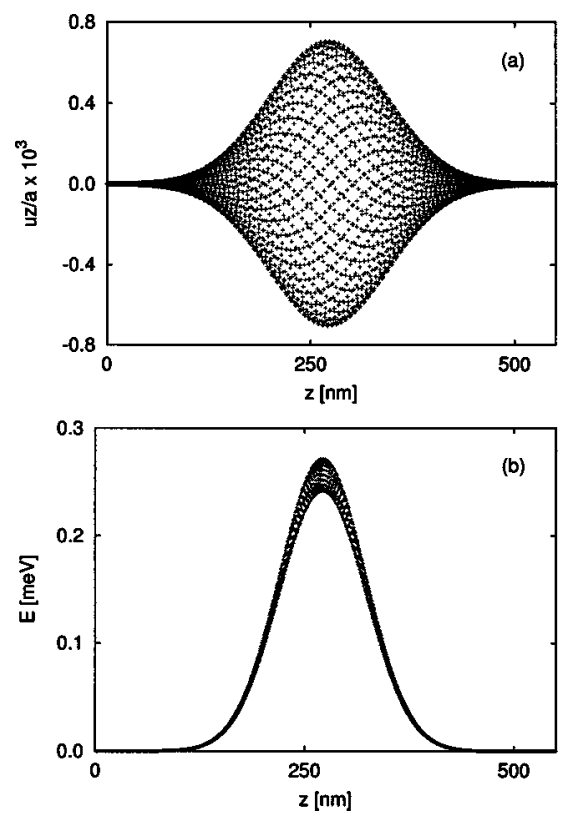

FIG. 4. (a) A localized phonon source or hotspot is created from a linear combination of the lattice vibrational modes, centered to have the required wave vector and polarization. The longitudinal displacements of individual atoms at different axial locations are shown in the figure. (b) The corresponding energy distribution is shown. The total energy in this hotspot is 2 $\mathrm{eV}$ and the energy density is $5 \times 10^{5} \mathrm{~J} / \mathrm{m}^{3}$.

\section{MODELING THE PHONON SOURCE IN A TRANSISTOR}

In this section we describe the actual hotspot in a device and discuss how we model it as a phonon wave packet in a molecular-dynamics computation. The hotspot in a device has a power density in the range of $5-50 \mathrm{~W} / \mu \mathrm{m}^{3}$ depending on the current density, and a full width at half maximum of about $10 \mathrm{~nm}$. The hotspot is located in the drain and is asymmetrical in shape as shown in Fig 1. The shape of the hotspot follows from the asymmetry in the electric field near the drain-channel interface. The electric field in a device peaks sharply toward the drain end of the channel. In the third direction, the hotspot extends throughout the width of the device. Neglecting end effects, the hotspot may be assumed to have an infinitely long axis in the third direction for the purpose of modeling.

In our study, we model the hotspot as a phonon wave packet that is localized in space using a Gaussian. We choose the longitudinal optical phonon, involved in a $g$-type electron scattering, as the focus for this study because nonzero group velocity optical modes have not been modeled in past studies on transistor hotspots. We note that other phonon modes can be just as easily studied. The wave packet used in this study is centered in wave vector space at $q=0.3[001] 2 \pi / a$, as follows from the previous section. The size and energy are adjusted to yield the desired hotspot. We multiply the atomic displacements associated with the initial phonon mode by a Gaussian to get the distribution in Fig. 4(a). The Gaussian width is about $32 \mathrm{~nm}$ for the wave packet shown. The initial energy distribution is related to the square of the displacements and is, thus, also a Gaussian as shown in Fig. 4(b). The energy density for this wave packet is approximately 
$5 \times 10^{5} \mathrm{~J} / \mathrm{m}^{3}$ which, given an average relaxation time of 0.1 ps for an electron-phonon scattering event, corresponds to a power density of $5 \mathrm{~W} / \mu \mathrm{m}^{3}$. The size of the source is comparable to the thermal phonon mean free path in silicon at room temperature, and thus allows investigation of subcontinuum transport. As far as the shape of the hotspot is concerned, the Gaussian profile is a good first approximation to the long-tailed but asymmetric hotspot in a transistor.

In comparing the simulated hotspot with the real hotspot, we note that there are several aspects that are not considered in this study, mainly due to computational constraints. First, as detailed above, the actual hotspot is constrained along the two directions in the plane of a device, whereas the wave packet is constrained in only one direction. This is described in greater detail in the following section. Second, the oxide interfaces and the dopants in the drain are neglected, both of which would be important scattering mechanisms for hotspot phonons. Third and most importantly, thermal phonons are not included in the simulation. Inclusion of any of these in the framework of molecular dynamics is particularly challenging. In the present work we have elected to study the anharmonic interactions in isolation. We have chosen to freeze out thermal phonons in the first set of simulations to understand the self-decay of hotspot phonons better. Thermal phonons will be included in future studies once the simpler picture is understood.

In the rest of this section we describe how we create a phonon wave packet for the MD simulations. We start with a linear combination of the eigenmodes of the bulk silicon crystal, described by the Stillinger-Weber ${ }^{28}$ potential. The eigenmodes themselves are obtained from harmonic lattice dynamics which is reviewed here in brief. Within the harmonic approximation, the equation of motion of the atoms can be written as ${ }^{13}$

$$
M_{k} \frac{\partial^{2}}{\partial t^{2}} u_{\alpha}\left(\begin{array}{l}
\ell \\
k
\end{array}\right)=-\sum_{\beta \ell^{\prime} k^{\prime}} \phi_{\alpha \beta}\left(\begin{array}{cc}
\ell & \ell^{\prime} \\
k & k^{\prime}
\end{array}\right) u_{\beta}\left(\begin{array}{l}
\ell^{\prime} \\
k^{\prime}
\end{array}\right),
$$

where $M_{k}$ is mass of the $k$ th atom in the $\ell$ th unit cell, $u$ is the displacement of the atom from its equilibrium position, $\alpha, \beta$ denote Cartesian vector components, and $\phi$ is the forceconstant matrix. Equation (1) is solved by introducing plane waves with wave vector $\mathbf{q}$, which decouples the equations for different wave vectors. This leads to the so-called dynamical matrix, D composed of $3 \times 3$ blocks, each block having elements

$$
\begin{aligned}
D_{\alpha \beta}\left(\begin{array}{c}
\mathbf{q} \\
k k^{\prime}
\end{array}\right)= & \frac{1}{\left(M_{k} M_{k^{\prime}}\right)^{\frac{1}{2}}} \sum_{\ell^{\prime}} \phi_{\alpha \beta}\left(\begin{array}{ll}
\ell & \ell^{\prime} \\
k & k^{\prime}
\end{array}\right) \\
& \times \exp \left[i \mathbf{q} \cdot\left\{\mathbf{R}\left(\begin{array}{l}
\ell^{\prime} \\
k^{\prime}
\end{array}\right)-\mathbf{R}\left(\begin{array}{l}
\ell \\
k
\end{array}\right)\right\}\right],
\end{aligned}
$$

where $\mathbf{R}$ is the position vector and $M$ is the mass of an atom. The next step is to diagonalize the dynamical matrix to yield its eigenvectors and eigenvalues. The normal mode frequency $\omega(\mathbf{q}, p)$ of the $p$ th branch is given by the square root of the corresponding eigenvalue of the dynamical matrix.
To localize the wave packet in space, we take the displacements of the optical mode with wave vector $\mathbf{q}_{0}$ and multiply it with a Gaussian ${ }^{20,21}$ to get

$$
\begin{aligned}
u_{\alpha}\left(\begin{array}{l}
\ell \\
k
\end{array}\right)= & A \epsilon_{k \alpha j}\left(\mathbf{q}_{0}\right) \exp \left[i \mathbf{q}_{0} \cdot\left(\mathbf{R}_{\ell}-\mathbf{R}_{0}\right)\right] \\
& \times \exp \left[-\frac{\left|\mathbf{R}_{\ell}-\mathbf{R}_{0}\right|^{2}}{\eta^{2}}\right],
\end{aligned}
$$

where $A$ is the amplitude of the wave packet, $\epsilon_{k \alpha j}$ is the polarization vector component for the branch $j$ at wave vector $\mathbf{q}_{0}$. This creates a wave packet that is centered in wave vector space at $\mathbf{q}_{0}$ and in geometrical space at $\mathbf{R}_{0}$. The spatial extent of the wave packet is set through the Gaussian width $\eta$. We set the initial atomic velocities such that the wave packet travels with a single group velocity. This requires that we know the eigenvector components which generate the displacements given by Eq. (3). The displacements of Eq. (3) can be expanded in terms of the eigenstates as

$$
u_{\alpha}\left(\begin{array}{l}
\ell \\
k
\end{array}\right)=\sum_{j \mathbf{q}} a_{j}(\mathbf{q}) \epsilon_{k \alpha j}(\mathbf{q}) \exp \left[i \mathbf{q} \cdot \mathbf{R}\left(\begin{array}{l}
\ell \\
k
\end{array}\right)\right] .
$$

The unknown expansion coefficients, $a_{j}(\mathbf{q})$, are found from

$$
a_{j}(\mathbf{q})=\sum_{k \ell \alpha} u_{\alpha}\left(\begin{array}{l}
\ell \\
k
\end{array}\right) \epsilon_{k \alpha j^{\dagger}}(\mathbf{q}) \exp \left[-i \mathbf{q} \cdot \mathbf{R}\left(\begin{array}{l}
\ell \\
k
\end{array}\right)\right]
$$

and finally the initial velocities are computed as

$$
v_{\alpha}\left(\begin{array}{l}
\ell \\
k
\end{array}\right)=\sum_{j \mathbf{q}} i \omega_{j}(\mathbf{q}) a_{j}(\mathbf{q}) \epsilon_{k \alpha j}(\mathbf{q}) \exp \left[i \mathbf{q} \cdot \mathbf{R}\left(\begin{array}{l}
\ell \\
k
\end{array}\right)\right] .
$$

With the above computations taken care of, the initial conditions for a MD simulation are fully described. The next section provides details of the MD computations.

\section{MD SIMULATIONS}

\section{A. Simulation cell}

Since we intend to compute decay mechanisms, we need to observe the dynamics over several hundred picoseconds, with the wave packet traveling about $1.5 \mathrm{~nm}$ per picosecond. Thus, in all of our work we use a simulation cell with threedimensional periodic boundary conditions. In choosing the specific dimensions of the supercell, a thin layered system is the obvious first choice since it is similar to an actual semiconductor device. Unfortunately, we found from preliminary simulations on a thin layer comprising $40 \times 40 \times 2$ unit cell that periodic boundary conditions resulted in the hotspot phonons interfering with each other after only a few picoseconds. In order to avoid this effect while still using a thin layer system, we would need to use a supercell with dimensions much too large for our computational capabilities. In view of these constraints, we instead chose a very long simulation cell, with a rather small cross section as described below. This permits the wave packet to propagate for several hundred picoseconds without spurious interference effects. This is described in greater detail in the next subsection. 


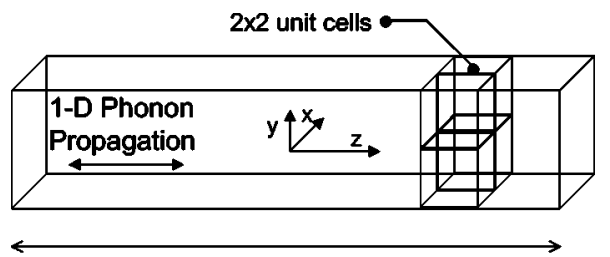

2000 unit cells $(\sim 1 \mu \mathrm{m})$

FIG. 5. The simulation is performed on a silicon super cell with 64000 atoms. The super cell has $2 \times 2$ units cells along the $x$ and $y$ directions as shown. There are 2000 unit cells in the axial $z$ direction corresponding to a length of approximately $1 \mu \mathrm{m}$. The boundaries are periodic along all three directions. The hotspot extends infinitely in the $x$ and $y$ directions and is a Gaussian along the $z$ direction

While this choice of geometry does not allow certain phonon decay channels, it does allow the most probable small-angle scattering events.

We chose the simulation cell to be 2000 unit cells long in the $z$ direction as shown in Fig. 5. Given the lattice parameter for silicon of $5.43 \mathrm{~A}^{\circ}$, this corresponds to a length of about $1 \mu \mathrm{m}$. By contrast, the simulation cell is only 2 unit cells wide in the $x$ and $y$ directions. Periodic boundary conditions along the $x$ and $y$ directions imply that the hotspot extends infinitely along these dimensions. Thus, the simulated hotspot is constrained only along the $z$ direction and resembles a sheet, as opposed to a device hotspot that is constrained along two directions and resembles a cylinder. The Gaussian profile, as shown in Fig. 4, is along the $z$ direction. Overall, the super cell contains 64000 atoms.

Before starting the MD, the phonon wave packet representing the hotspot is embedded in the super cell. The displacements and velocities are prescribed as discussed in the previous section. A microcanonical ensemble, i.e., constantNVE ensemble, ${ }^{29}$ is specified and the system is evolved in time using MD. Postprocessing involves decomposing the atomic displacements into the eigenmodes to find phonons of different polarizations.

\section{B. Procedure to remove the negative strain field}

Considerable care must be taken to avoid spurious effects from the strain fields associated with the hotspot. Associated with the localized phonon wave packet is a localized region of positive strain. This may be understood from the Grüneisen theory of thermal expansion, which relates the thermal expansion coefficient to the dependence of the phonon frequencies on lattice strain. For simulations in which the volume of the simulation cell is fixed, the positive strain created by the introduction of the hotspot will be compensated by a negative strain; this appears as a negative strain field that propagates through the system at the speed of sound. Due to the finite size of the simulation cell and the use of periodic border conditions, this negative strain region can interact with the positive strain region localized at the hotspot. This is evident from Fig. 6 where the strain field for a typical simulation is shown at various times. The positive strain in each snapshot corresponds to the location of the optical phonon hotspot.

As the final snapshot in Fig. 6 shows, this interaction acts to cancel the positive strain region localized at the

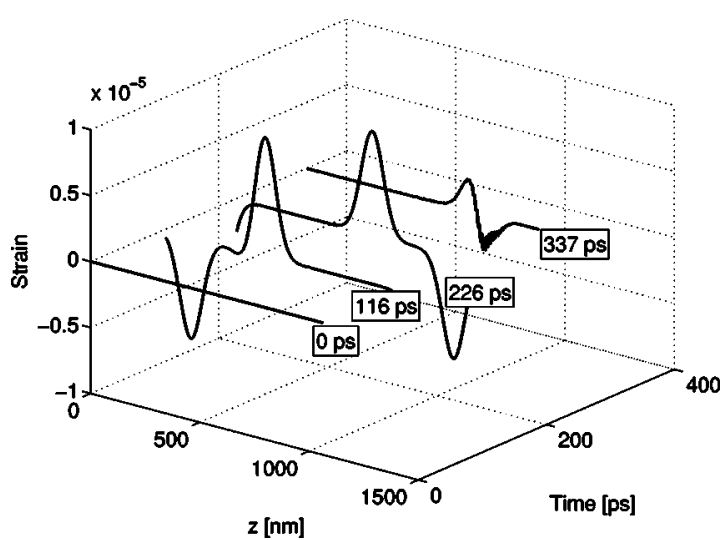

FIG. 6. The strain in the system is shown as a function of time for an initial maximum amplitude of $0.001 a_{0}$ where $a_{0}$ is the lattice parameter for silicon. The local positive strain at the hotspot is canceled by the negative strain outside at 337 ps which also causes the collapse of the hotspot.

hotspot. The last snapshot in Fig. 6 shows that the local positive strain, which is approximately $10^{-5}$ in the previous snapshots, is about an order of magnitude lower from the cancellation. This final snapshot also corresponds to a collapse of the hotspot, suggesting the possibility that the scattering is being precipitated by the interaction with the negative strain region. If this is indeed the case, then in Fig. 6 we are not observing intrinsic hotspot decay, but rather decay caused by the interaction with the negative strain region.

This effect will also be present in more realistic constant pressure simulations. The constant pressure MD algorithm will introduce a homogeneous expansion or contraction of the simulation cell in response to the presence of an inhomogeneity at the hotspot. Over an extended period of time, this would allow, at least in principle, the correct inhomogeneous strain field to be produced, though for any reasonably sized system the negative and positive strain field regions would interact with each other first.

To determine the intrinsic decay mechanisms of a localized hotspot, it is necessary to devise a simulation method that will eliminate the negative strain region. The procedure that we have employed here involves two steps. The first step is to perform an MD simulation for a relatively short simulation time (approximately $66 \mathrm{ps)}$ ). In this time, the hotspot has had time to create the localized positive lattice strain, but the compensating negative strain region has not had sufficient time to propagate and interact with the hotspot. This time corresponds to the displacements shown in Fig. 7(a). At the end of this first step, we save the final atomic displacements and velocities.

The second step is to modify the displacements in such a way as to remove the negative strain region. We first choose two points on either side of the hotspot that are in regions of zero strain. In the simulation shown in Fig. 7(a), we chose these points at $z_{\ell}=450 \mathrm{~nm}$ and $z_{r}=980 \mathrm{~nm}$. We next create the set of atomic displacements shown in Fig. 7(b). For all atoms inside the hotspot region (i.e., with positions in between $z_{\ell}$ and $z_{r}$ ) we directly use the atomic displacements and velocities from the first simulation. For atoms outside this region, we assign atomic displacements such that there is no lattice strain for the regions $z<z_{\ell}$ and $z>z_{r}$. In addition, 

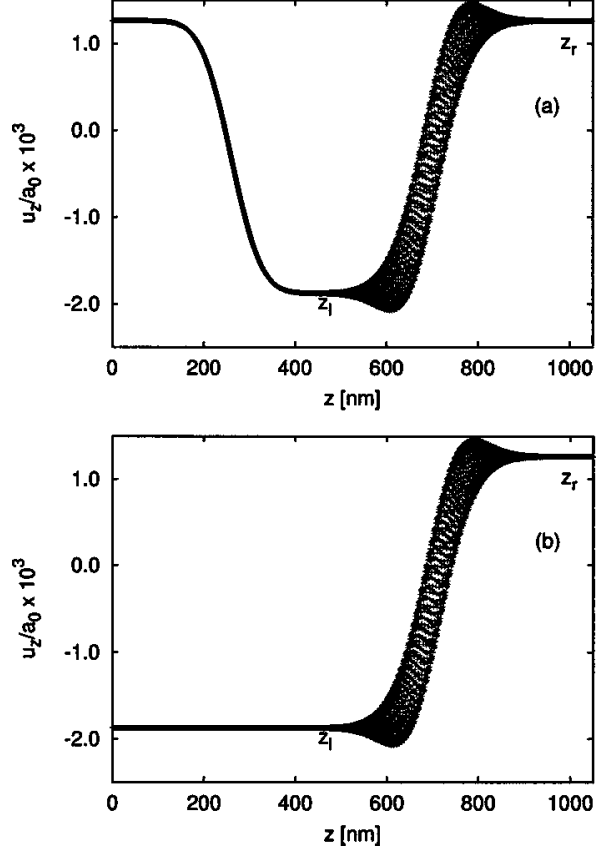

FIG. 7. (a) The axial displacements are shown at 66 ps into the first MD run. The hotspot is centered around $700 \mathrm{~nm}$ with large amplitude long wavelength modes outside. (b) The hotspot is carved out of this snapshot by setting the displacements outside the hotspot to zero. The strain adjusted system has an expanded lattice and has zero strain outside the hotspot. The second MD simulation is started with this initial condition.

we set the velocity of each atom outside of the hotspot region to zero. Finally, we increase the length of the simulation cell to allow for the positive strain at the hotspot. This set of atomic displacements and velocities are then used as input for another MD simulation.

In the second step of the MD procedure, the hotspot still propagates along with the positive strain region, but now the spurious negative strain region is absent. This is shown in Fig. 8. In this system the wave packet still decays but after a significantly longer time. For the case of a $2 \mathrm{eV}$ hotspot, we find that the wave packet begins to decay after 431 ps of simulation without the negative strain region, as compared with $337 \mathrm{ps}$ in the presence of the negative strain region. The

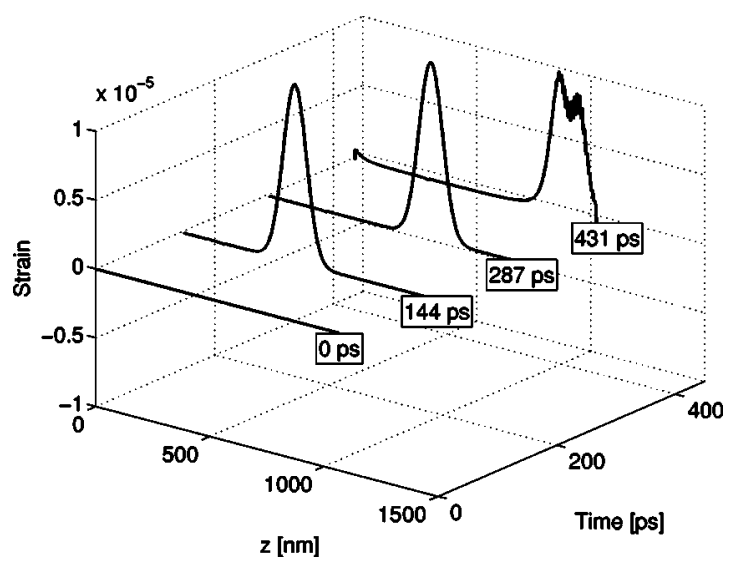

FIG. 8. The strain in the system for the two-step MD procedure is shown. The hotspot is the same as that in Fig. 6. Without the negative strain region, the wave packet has a longer lifetime, of about 431 ps. This is to be compared with 337 ps in Fig. 6. time to decay is computed by monitoring the energy in the excited mode. Once the scattering starts, the energy associated with the mode shows an exponential decay. These results demonstrate that the negative lattice strain was indeed initiating scattering in the first simulation.

In order to confirm that the scattering we observe in the two-step procedure is an intrinsic anharmonic effect, we have performed a reference simulation in which the initial amplitudes of displacements inside the hotspot are so low that there are no significant anharmonic interactions. If anharmonicity alone causes scattering in our MD simulations, then this case would show no scattering. We performed simulations on a "hotspot" where the maximum initial displacement is only $1 / 100$ th of that of a $2 \mathrm{eV}$ hotspot; i.e., the total energy in the hotspot is approximately $0.2 \mathrm{meV}$. After a very long simulation of $1.82 \mathrm{~ns}$, we find no evidence of scattering. This is strong evidence that the scattering we observe in our simulations is indeed due to anharmonic phonon-phonon interactions.

\section{RESULTS AND DISCUSSION}

In this section we use the MD procedure described above to compare anharmonic phonon scattering at hotspots with two different energy densities. The spatial extent of the hotspot is the same in both cases and corresponds to that shown in Fig. 4. The first hotspot has an energy of $2 \mathrm{eV}$ and corresponds to a moderately high energy density of $5 \mathrm{~W} / \mu \mathrm{m}^{3}$. The second hotspot has an energy of $50 \mathrm{eV}$ and corresponds to an energy density of $125 \mathrm{~W} / \mu \mathrm{m}^{3}$. For reference, the hotspot in the device shown in Fig. 1 has a peak energy density of about $65 \mathrm{~W} / \mu \mathrm{m}^{3}$.

Since we have frozen out thermal phonons in our calculations, the hotspots have extended lifetimes, over $400 \mathrm{ps}$ for the $2 \mathrm{eV}$ case and over $100 \mathrm{ps}$ for the $50 \mathrm{eV}$ case. During this period, there is spontaneous scattering into low frequency modes due to anharmonicity but this reduces the energy in the wave packet by less than $1 \%$. However, interactions of the wave packet with this background of low frequency modes triggers the eventual scattering of the wave packet. This second scattering is characterized by an exponential decrease in energy at the hotspot. The various decay modes during this event have energies comparable to that of the initial wave packet and are clearly visible in Fig. 9 which shows snapshots of the scattering process in real space for a $2 \mathrm{eV}$ hotspot.

In order to analyze the scattering process, we first compute the projections of the atomic displacements onto the eigenvectors of each mode. This is similar to the analysis of the initial displacements as detailed in Eq. (4). We further use the projections to compute the energy associated with each normal mode at various times. From this, we can deduce which phonons are created or annihilated over time. Figure 10 shows the decay in energy for two wave packets. The lower energy wave packet has a maximum amplitude of $0.001 a_{0}$, where $a_{0}$ refers to the lattice parameter of silicon, while the higher amplitude wave packet has a maximum amplitude of $0.005 a_{0}$. Scattering is initiated in the $50 \mathrm{eV}$ case after $120 \mathrm{ps}$ and in the $2 \mathrm{eV}$ case after 431 ps. As discussed 

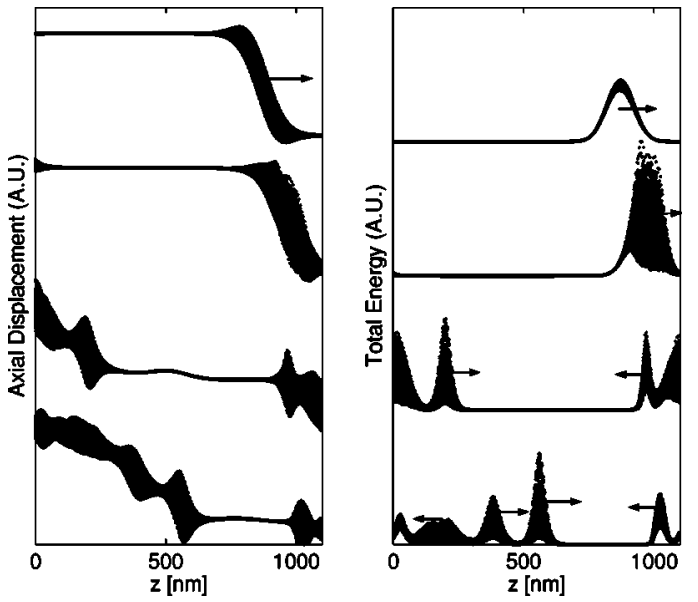

FIG. 9. Snapshots of anharmonic phonon scattering at a $2 \mathrm{eV}$ hotspot, as observed in the strain corrected MD, are shown. The axial displacements are plotted on the left and the corresponding energy distribution in real space is plotted on the right. The $x$ axis shows the axial location. The snapshot at the top is taken just before scattering is initiated. As scattering proceeds, phonons of various modes are created, corresponding to the lumps on the left and the peaks on the right, and propagate from the hotspot at different group velocities. The arrows indicate the direction of propagation.

above, the decay in the energy of the wave packet is not exponential over this time period as is observed when thermal phonons are present (as in Ref. 18, for example). There is some scattering into low frequency modes, corresponding to a less than $1 \%$ decrease in energy of the wave packet. After an extended period, anharmonic interactions with these modes become strong enough to precipitate scattering at the hotspot. Once the scattering is initiated, the decay in energy is exponential with superposed oscillations. Though the oscillations appear to be present only for the lower excitation case in Fig. 10, they are, in fact, present at both excitations. It is merely that the larger decay time for the lower excitation makes the oscillations markedly visible. The projection of the atomic displacements onto the eigenvector of the initially excited LO mode has an exponentially decaying sinusoidal form as a function of time. ${ }^{18}$ These sinusoidal oscillations are manifest in the energy as well since the energy is related to the square of the projection. We find the decay constant for the exponential part of the curve to be 16 ps for the $50 \mathrm{eV}$ case and $79 \mathrm{ps}$ for the $2 \mathrm{eV}$ case. These relaxation times are almost inversely proportional to the initial amplitudes of displacement, $0.005 a_{0}$ and $0.001 a_{0}$, respectively, consistent with the theoretical predictions from perturbation theory. ${ }^{16}$

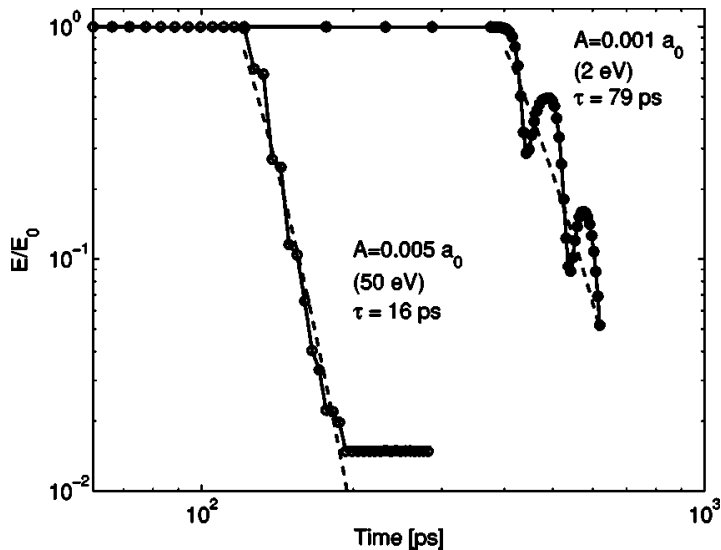

FIG. 10. The energy in the entire system associated with the longitudinal optical phonon mode is shown as a function of time. $E_{0}$ refers to the initial energy of the hotspot, which is $50 \mathrm{eV}$ for the curve on the left and $2 \mathrm{eV}$ for the curve on the right. The corresponding amplitudes are also given in terms of the lattice spacing, $a_{0}$, and $\tau$ is the decay constant in the exponential part of the curve.

Further, it is illustrative to compare these numbers with optical phonon lifetimes measured in Raman scattering experiments, even though the phonon involved in Raman scattering is typically the zone-center optical mode. The zone-center optical mode in silicon has a lifetime of approximately $55 \mathrm{ps,}$ corresponding to a full width at half maximum of $1.24 \mathrm{~cm}^{-1}$, at low temperatures. ${ }^{27}$ The lifetime at room temperature has been measured to be in the range $10-25 \mathrm{ps}^{22,27}$ We note here that since the power density is not reported in these studies, we provide these figures only to show an order of magnitude comparison with our study.

We have analyzed the decay spectrum, finding that the scattering mechanism for hotspot LO phonons involves multiple phonon frequencies and polarizations. We note here that since we compute the energy in each phonon mode at different times, we know the frequency, wave vector, and polarization of modes that are annihilated or created. Figures 11(a) and 11(b) show snapshots of the spectrum for the 2 and the $50 \mathrm{eV}$ hotspots, respectively. The initial decay channel is the same in both cases but an additional decay channel is subsequently created in the higher energy case. The first stage involved decay into two dominant frequencies, as shown in Fig. 12. The zone-edge transverse acoustic phonons with frequencies centered at $\nu_{1}=7.82 \mathrm{THz}$ dominate in terms of overall energy. Longitudinal acoustic phonons with fre-

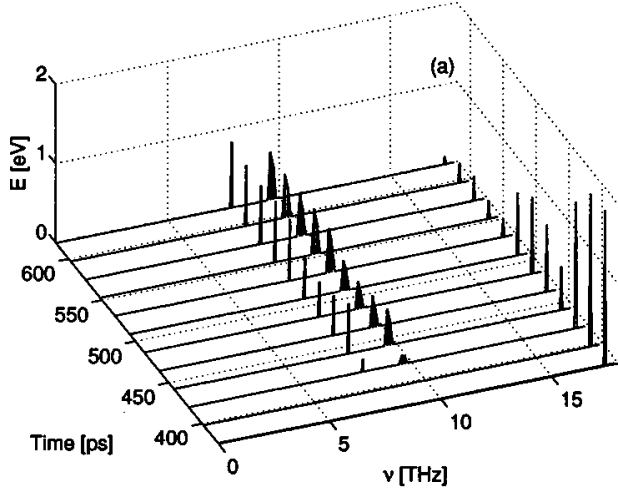

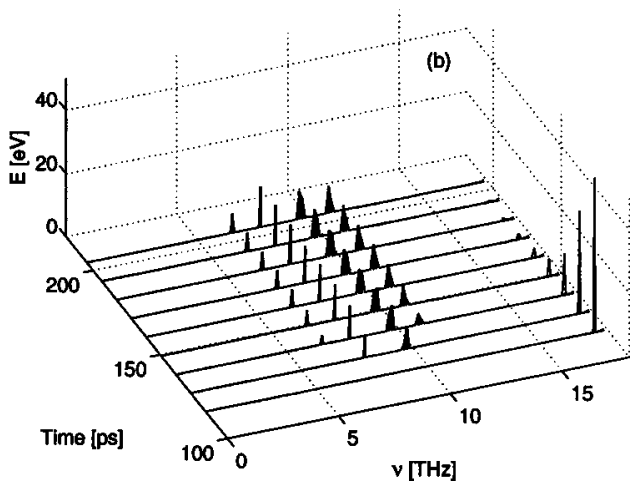

FIG. 11. The distribution of energy in the phonon spectrum is shown at various times for a hotspot with energy (a) $2 \mathrm{eV}$ energy, and (b) $50 \mathrm{eV}$. The higher energy results in accelerated multiple decay stages. The second stage decay is not observed in the lower energy case up to 620 ps into the simulation. 

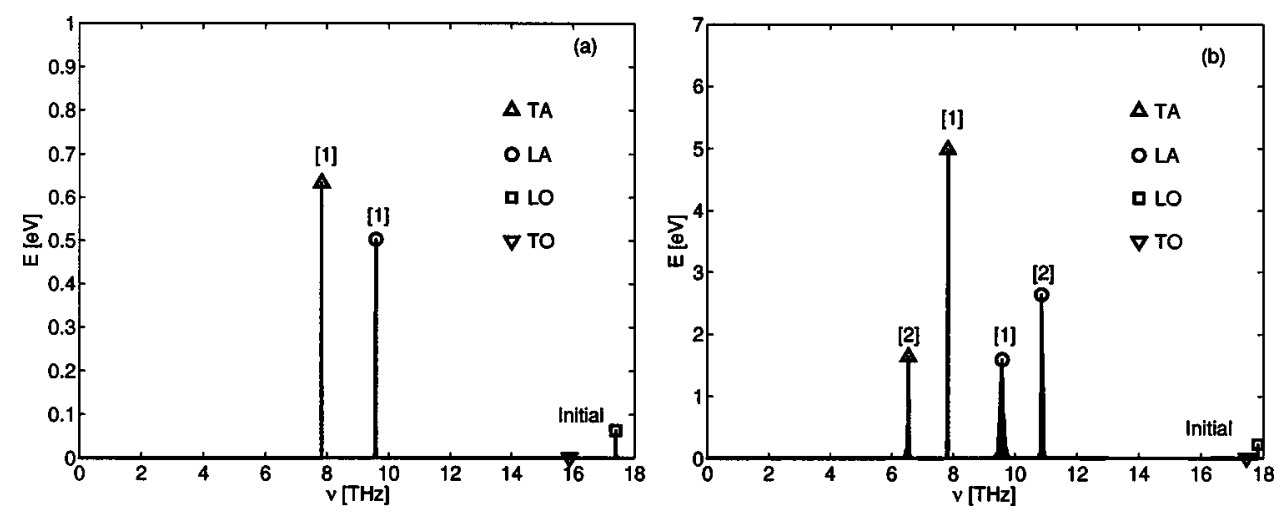

FIG. 12. The distribution of energy for various polarizations is shown at (a) $620 \mathrm{ps}$ for a $2 \mathrm{eV}$ hotspot and (b) at $255 \mathrm{ps}$ for a $50 \mathrm{eV}$ hotspot. The first decay channel, labeled 1 , is a three phonon process of the form $L O \rightarrow L A$ $+T A$ in both cases. A second process, labeled 2, of the same form is observed in the $50 \mathrm{eV}$ case. Zone-edge TA phonons are found to be dominant irrespective of the initial energy density. Table I lists the wave vectors involved in the processes. quencies centered at $\nu_{2}=9.58 \mathrm{THz}$ are created as well. The initial LO wave packet shows a spread in frequencies, with the center at $\nu_{0}=17.40 \mathrm{THz}$. The frequencies sum up to a three phonon processes of the type $\nu_{0} \rightarrow \nu_{1}+\nu_{2}$. To rule out higher order processes, we have characterized the spread in frequencies. Figure 13 shows the spread in frequency around $\nu_{0}$ in the LO wave packet just before the principal scattering event is initiated. There is a slight broadening towards lower frequencies with time due to anharmonic scattering into low frequency modes as mentioned above. Also shown is the spread in frequency around $\nu_{1}$ after the scattering. An asymmetric Lorentzian can be used as an envelope function in both cases. The spread around $\nu_{2}$ is similarly contained within the envelope function. Thus, a three phonon process of the form $\nu_{0} \rightarrow \nu_{1}+\nu_{2}$ explains the presence of the TA and LA frequencies. The spread in the frequencies of the decayed modes is merely due to the spread in the frequency of the initial wave packet. An accelerated second stage decay occurs in the $50 \mathrm{eV}$ case as shown in Figs. 11(b) and 12(b). Transverse acoustic phonons with frequencies centered at $\nu_{3}=6.55 \mathrm{THz}$ and longitudinal acoustic phonons with frequencies centered at $\nu_{4}=10.85 \mathrm{THz}$ show up in this case. This decay is, once again, a three phonon process of the type
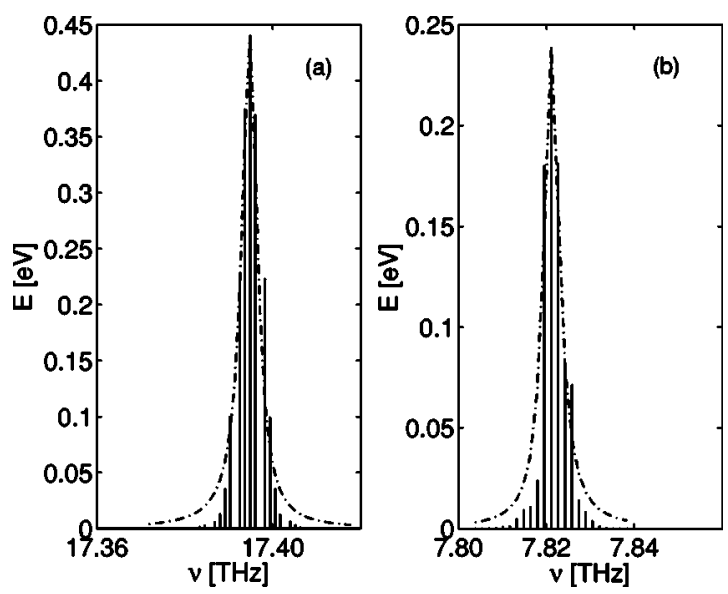

FIG. 13. The frequency spread in the LO wave packet, prior to scattering, is shown on the left. An asymmetric Lorentzian, with a total width of 0.009 $\mathrm{THz}$, is plotted as an enveloping function. The spread in TA frequencies, after scattering, is shown on the right. The spread is enveloped by the same Lorentzian, with a mirror reflection around the center. This strongly suggests that the spread in frequencies of the decayed phonons is due to the frequency spread in the initial wave packet alone and not due to higher order processes. $\nu_{0} \rightarrow \nu_{3}+\nu_{4}$. We have checked momentum conservation in each case and find all the processes to be normal processes. We list the wave vectors involved in the two decay processes in Table I. Several wave vector combinations are possible for each of the two processes, but in each case they involve three phonons only. We note here that we do not think that the second stage of decay is exclusive to the higher excitation energy only. All decay processes are accelerated at the higher excitation due to the increased anharmonic contribution to the scattering rate. Hence, we are able to observe what would otherwise be an extremely slow second stage decay, within the time frame of our simulations.

Thus, at both the energy densities, three phonon processes of the form $L O \rightarrow L A+T A$ are involved. This is consistent with previous $a b$ initio perturbation calculations ${ }^{15}$ that show that the zone-center LO mode in silicon has a $94 \%$ probability for decaying into LA and TA modes. Debernardi and co-workers, ${ }^{15}$ explain why this decay channel is favored by defining a frequency-resolved final state spectra, $\gamma(\omega)$, which is the probability per unit time that a given LO mode, with frequency $\omega_{0}$, decays into a mode with frequency $\omega$ and another mode with frequency $\omega_{0}-\omega$. In the case of silicon, the overlap of the final state spectra with the phonon density of states is maximum for a TA and an LA mode.

The observation that zone-edge TA phonons are created near the hotspot irrespective of the energy density further suggests that the phonon hydrodynamic equations used in coupled electro-thermal simulations ${ }^{2,9}$ may need to be revised. According to these equations, the heat transferred to the lattice by the scattering of hot electrons is not conducted out of the hotspot until optical phonons decay into acoustic phonons. Subsequent to the decay, phonons conduct the heat away from the hotspot at acoustic velocity. The present study shows that at least at low temperatures, heat conduction is

TABLE I. Conservation of energy and momentum for the observed three phonon processes

\begin{tabular}{cccccc}
\hline \hline & Initial & \multicolumn{2}{c}{ First decay } & \multicolumn{2}{c}{ Second decay } \\
\hline & LO & LA1 & TA1 & LA2 & TA2 \\
\hline$q_{x} /\left(2 \pi / a_{0}\right)$ & 0.0 & $0.0, \pm 0.5$ & $0.0, \mp 0.5$ & $0.0, \pm 0.5$ & $0.0, \mp 0.5$ \\
$q_{y} /\left(2 \pi / a_{0}\right)$ & 0.0 & $\pm 0.5,0.0$ & $\mp 0.5,0.0$ & $\pm 0.5,0.0$ & $\mp 0.5,0.0$ \\
$q_{z} /\left(2 \pi / a_{0}\right)$ & 0.3 & 0.85 & -0.55 & 0.75 & -0.45 \\
$\nu(\mathrm{THz})$ & 17.40 & 9.58 & 7.82 & 10.85 & 6.55 \\
\hline \hline
\end{tabular}


impeded after the optical modes scatter. The effect at room temperature remains to be investigated.

\section{CONCLUDING REMARKS}

In this work we have developed a methodology to characterize the propagation and anharmonic scattering of high energy density optical phonons by MD simulation. We found that the process of embedding the wave packet requires special care to avoid spurious strain effects. A two-step procedure allows us to overcome this problem and observe the anharmonic self-scattering of the hotspot. We reported the scattering times for longitudinal optical phonons, involved in $g$-process intervalley transition in silicon, for both moderate and high energy densities and showed that the decay involves three phonon processes of the form $L O \rightarrow L A+T A$, consistent with predictions from previous $a b$ initio calculations on zone-center optical phonons. The zone-edge transverse acoustic phonon dominates in both cases. A second stage of decay is observed when the initial amplitude of excitation is high.

This is a direct observation of phonon decay processes for the nonequilibrium conditions corresponding to those in a transistor. While phonon decay channels are likely to change in the presence of $300 \mathrm{~K}$ thermal phonons, the method described in this article can be extended to include the background phonons. The authors are currently investigating this problem. The scattering rates obtained here can be used in less computationally intensive models based on the phonon BTE that are more useful in direct simulation of semiconductor devices.

\section{ACKNOWLEDGMENTS}

S.S. and K.E.G. thank the Semiconductor Research Corporation for support through task 1043. S.S. is grateful to the Powell foundation for sponsoring a Stanford Graduate Fellowship. The work of P.K.S. and S.R.P. at Argonne National Laboratory was supported by the U.S. Department of Energy, Office of Science under Contract No. W-31-109-Eng-38. S.S. thanks Eric Pop at Stanford University for the MEDICI input deck used in Fig. 1 and for many helpful discussions on electron-phonon scattering.

${ }^{1}$ D. G. Cahill, W. K. Ford, K. E. Goodson, G. D. Mahan, A. Majumdar, H. J. Maris, R. Merlin, and S. R. Phillpot, J. Appl. Phys. 93, 793 (2003).

${ }^{2}$ A. Majumdar, K. Fushinobo, and K. Hijikata, J. Appl. Phys. 77, 6686 (1995).

${ }^{3}$ International Technology Roadmap for Semiconductors (ITRS), 2003 ed., URL http://public.itrs.net (2003).

${ }^{4}$ P. G. Sverdrup, Y. S. Ju, and K. E. Goodson, J. Heat Transfer 123, 130 (2001).

${ }^{5}$ S. V. J. Narumanchi, J. Y. Murthy, and C. H. Amon, J. Heat Transfer 125, 896 (2003).

${ }^{6}$ G. Chen, J. Heat Transfer 118, 539 (1996).

${ }^{7}$ S. Sinha and K. E. Goodson, 12th International Heat Transfer Conference, Grenoble, France, 18-23 August, 2002.

${ }^{8}$ P. G. Sverdrup, S. Sinha, M. Asheghi, U. Srinivasan, and K. E. Goodson, Appl. Phys. Lett. 78, 3331 (2001).

${ }^{9}$ J. Lai and A. Majumdar, J. Appl. Phys. 79, 7353 (1996).

${ }^{10}$ D. V. Kazakovtsev and I. B. Levinson, Sov. Phys. JETP 61, 1318 (1985).

${ }^{11}$ Y. J. Han and P. G. Klemens, Phys. Rev. B 486033 (1993).

${ }^{12}$ M. G. Holland, Phys. Rev. 1322461 (1963).

${ }^{13}$ M. Born and K. Huang, Dynamical Theory of Crystal Lattices (Clarendon, Oxford, 1954).

${ }^{14}$ A. A. Maradudin and A. E. Fein, Phys. Rev. 128, 2589 (1962).

${ }^{15}$ A. Debernardi, S. Baroni, and E. Molinari, Phys. Rev. Lett. 75, 1819 (1995).

${ }^{16}$ J. Fabian and P. B. Allen, Phys. Rev. Lett. 77, 3839 (1996).

${ }^{17}$ S. R. Bickham and J. L. Feldman, Phys. Rev. B 57, 12234 (1998)

${ }^{18}$ C. Oligschleger and J. C. Schön, Phys. Rev. B 59, 4125 (1999).

${ }^{19}$ A. J. C. Ladd, B. Moran, and W. G. Hoover, Phys. Rev. B 34, 5058 (1986).

${ }^{20}$ P. K. Schelling, S. R. Phillpot, and P. Keblinski, Appl. Phys. Lett. 80, 2484 (2002)

${ }^{21}$ P. K. Schelling and S. R. Phillpot, J. Appl. Phys. 93, 5377 (2003).

${ }^{22}$ D. K. Ferry, Semiconductor Transport (Taylor \& Francis, New York, 2000).

${ }^{23}$ E. Pop, R. W. Dutton, and K. E. Goodson, in International Conference on Simulation of Semiconductor Processes and Devices (SISPAD), Cambridge, MA, 3-5 September 2003.

${ }^{24}$ D. Long, Phys. Rev. 120, 2024 (1960).

${ }^{25}$ D. K. Ferry, Phys. Rev. B 14, 1605 (1976).

${ }^{26}$ B. K. Ridley, Quantum Processes in Semiconductors (Clarendon, Oxford, 1999).

${ }^{27}$ J. Menèndez and M. Cardona, Phys. Rev. B 29, 2051 (1984).

${ }^{28}$ F. H. Stillinger and T. A. Weber, Phys. Rev. B 31, 5262 (1985).

${ }^{29}$ M. P. Allen and D. J. Tildesley, Computer Simulation of Liquids (Clarendon, Oxford, 1987). 